\title{
Research and implementation of Highcharts in patent map Shilong Guo ${ }^{1, a}$, Yong Wang ${ }^{2, b}$
}

\author{
${ }^{1}$ School of Computer,Guangdong University of Technology, Guangzhou, 51006, China \\ ${ }^{2}$ School of Computer, Guangdong University of Technology, Guangzhou, 51006, China \\ aemail: 736921396@qq.com, bemail: 13928887919@126.com
}

Key word: patent map; Highcharts; patent analysis; production software; analysis technology

\begin{abstract}
The B/S(Browser/Server) network structure plays an important role in computer architecture today, and it has become increasingly important to display the patent map in the browser, currently on the market circulation of a large number of patent map analysis software, but are generally very expensive and is not conducive to the study of small enterprises, organizations and individuals. Research and application of Highcharts technology in the patent map, and through the examples to illustrate the advantage of Highcharts technology in the browser display of patent map.
\end{abstract}

\section{Introduction}

Patent map (Map Patent) is a kind of patent analysis method and expression form, according to the deep mining and analysis of the economic information, technical information and legal information contained in the patent data, the hidden in the patent data of a large number of complex information through a variety of visual intuitive graphic form to express, similar to the function of the map guide[1]. Most of the patent map making tools are to be charged and expensive, for example, the software of PatMap(only for internal use), the software of Patent Guider[2] [3] and so on.

\section{Overall system design}

System target.Collect data from the patent database.Datasource from CNKI.

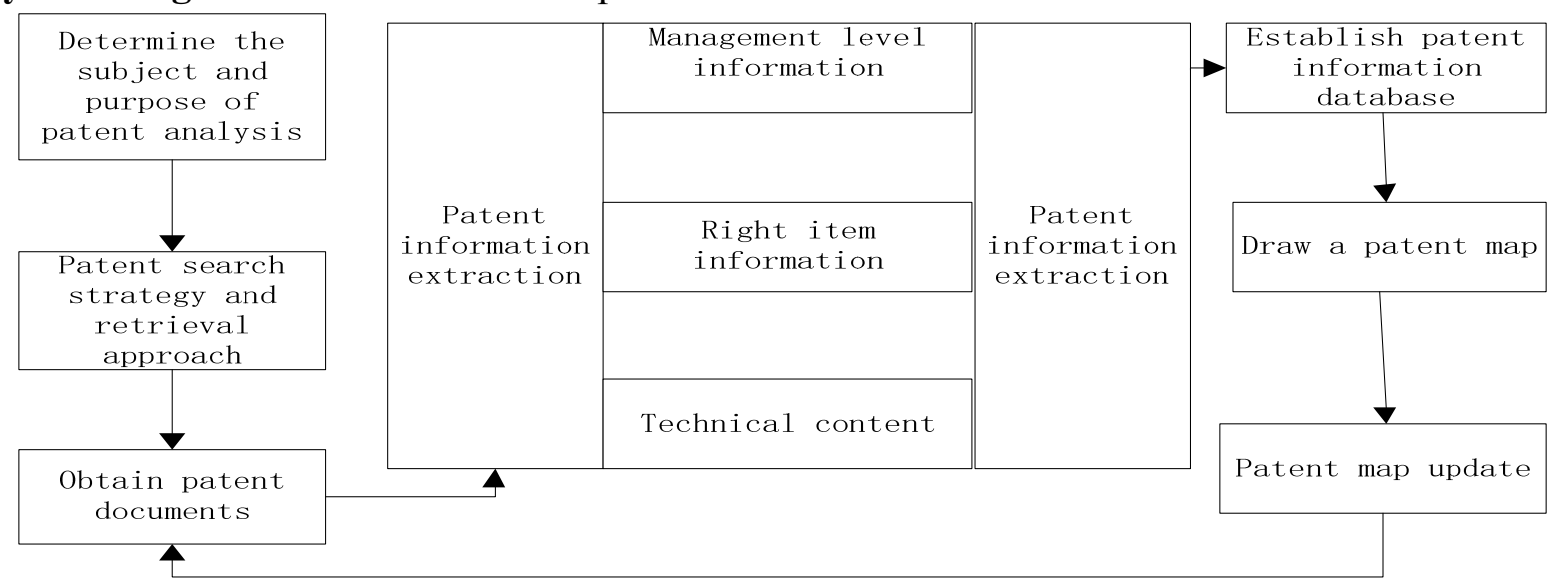

Fig. 1. Making process of patent map 
System framework. The System framework is shown in Figure 2

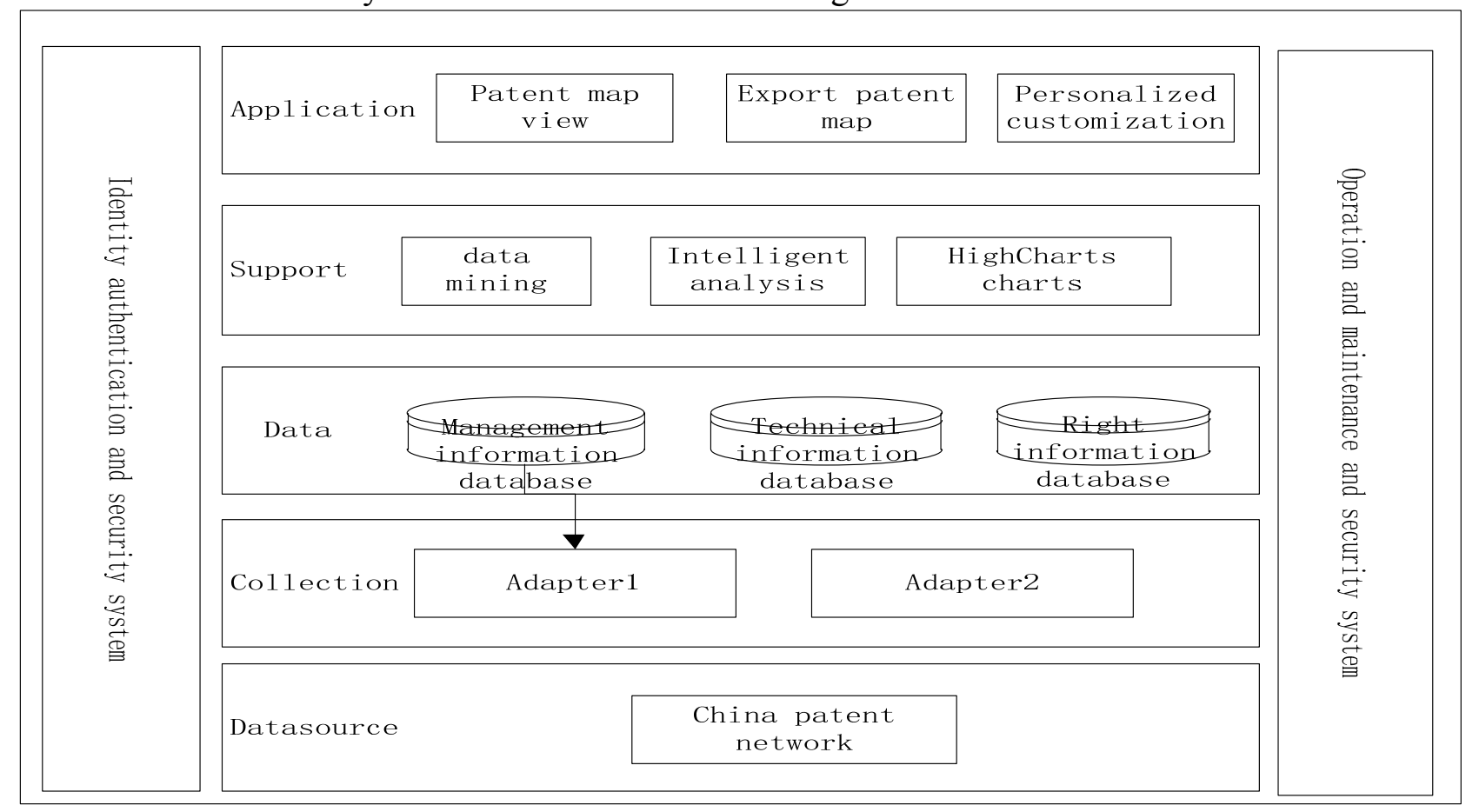

Fig. 2. System framework

\section{System database and function design}

Database design. The database design is very important for the presentation of the patent map, and according to different analysis purpose and focus, the current patent map can be divided into 3 categories: ManagementPM、TechnicalPM、ClaimPM.

System function design. The function module of this system is shown in Figure 3.

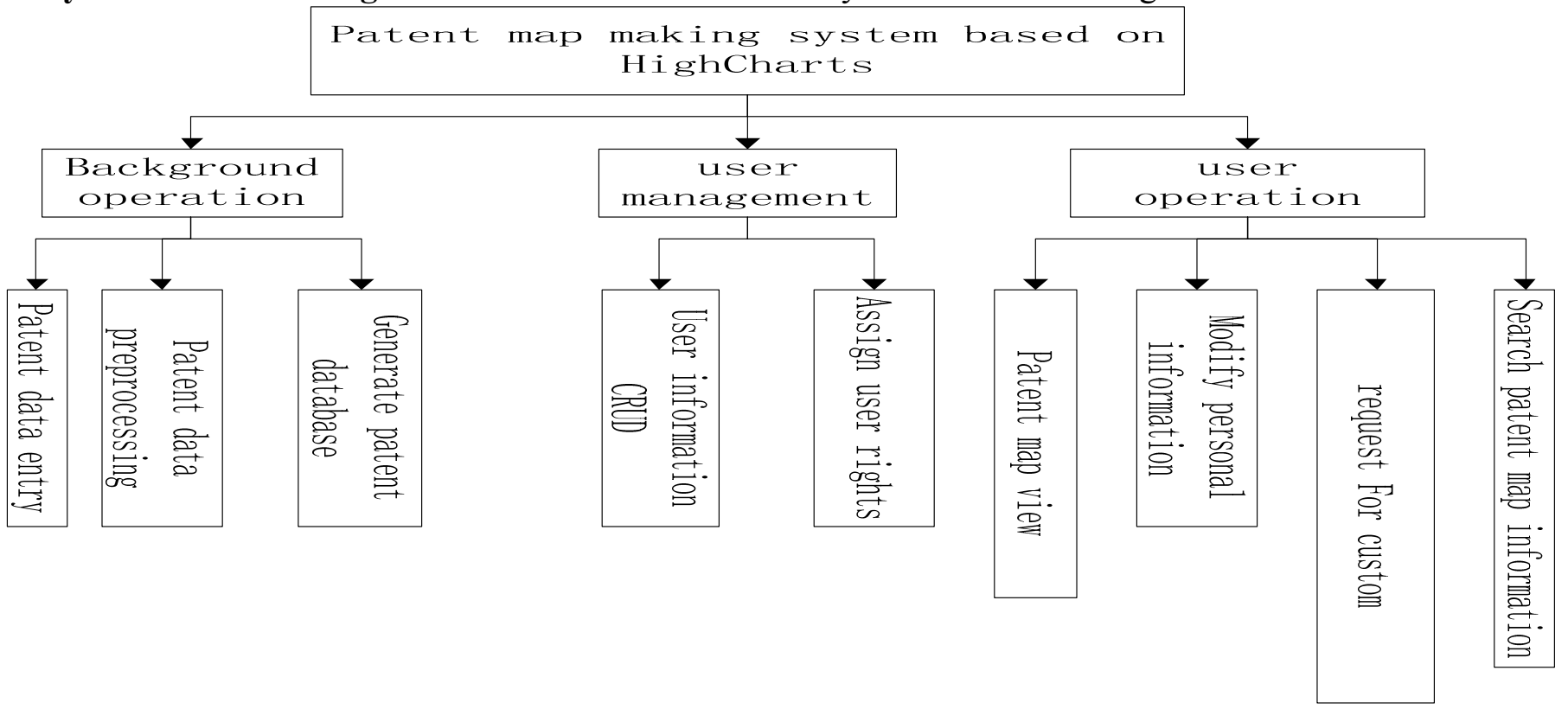

Fig. 3. Design of System functions 


\section{Key technology}

Text data preprocessing. Patent text data preprocessing process[4] shown in Figure 4.

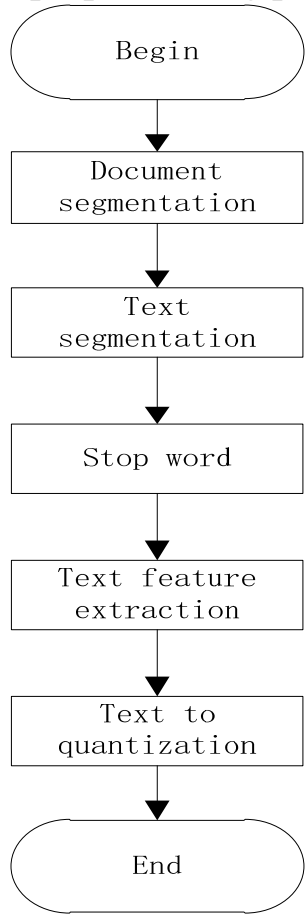

Fig. 4. Patent text data pretreatment process

Data mining. For patent data, especially patent technical information, data mining technology can be used to find the link between the patent information, which is used for enterprises and individuals. However, the huge amount of patent data information is often 10 million level, if the level of ten million level of patent data mining analysis and prediction is very difficult. Data mining plays an important role in information extraction and decision making.

K-Means algorithm. The specific process of K-means algorithm is described as follows: (1) From a given sample data from randomly selected k data points as the initial cluster centers; (2) Calculating data from each data to the distance of the $\mathrm{K}$ cluster centers and each data point is assigned away from the nearest center on behalf of the cluster; (3) To calculate the average of all data points in each cluster as a new center of each cluster; (4) To determine whether or not the clustering criterion function convergence or clustering centers and the last is exactly the same, if there is no change to the convergence or central point, is the end of the algorithm, output the clustering result, otherwise go to step (2)[5][6].

\section{Operation result}

The number distribution of patents in the year of 2003-2014 using MIMO Technology. From 2003 to 2014, the number of patents for the use of MIMO technology as shown in Figure 5. As can be seen in 2003 and 2004 for MIMO technology patent development slow, from the beginning of 2005 increased rapidly, to 2012 patent open up to the amount reached its peak, the number in 2013 began tended to be stable and that for MIMO technology patent research almost reached the saturated state, the individual or organization should find another patent $R \& D$ points. 


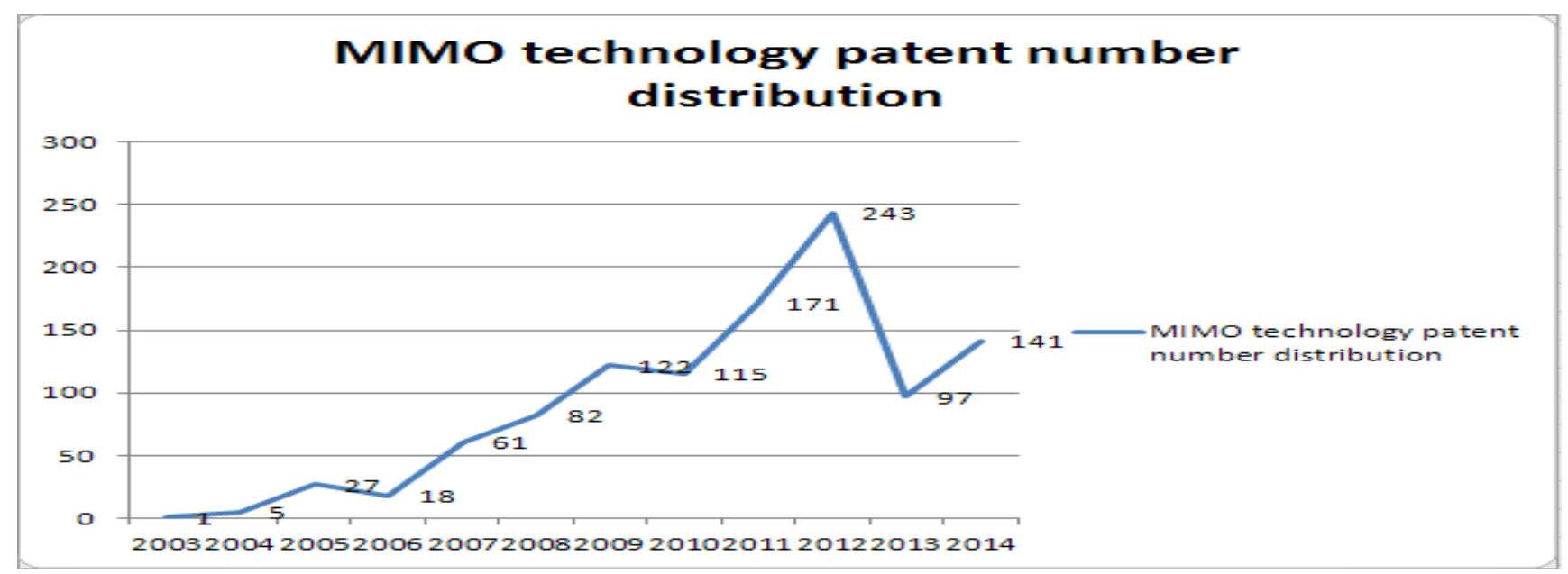

Fig. 5. MIMO technology patents quantity distribution by years

\section{Conclusions}

For patent map analysis software, although there are many charges on the market, is not conducive to small organizations and individuals on the patent map of the study, but also for the WEB end real-time display patent map is a limitation. This paper shows the application of HighCharts in patent map, it can be seen that HighCharts has a significant advantage in WEB end real-time display patent map, and it is suitable for small organizations and individuals to study the patent map.

\section{Acknowledgment}

In this paper, the research was sponsored by Guangdong Province, the Ministry of education, the combination of production and research project(No. 2012B040500005).

\section{References}

[1]ZHANG Xian, GAO Lidan, TANG Chuan,et al. Research and application of the patent map[J]. JOURNAL OF INFORMATION ,2007,26(11):22-25(in Chinese).

[2]WU Xinyin, LIU Ping, Qi Changwen.Study and analysis of manufacturing patent map[J].ELECTRONICS INTELLECTUAL PROPERTY,2003(11):20-23(in Chinese).

[3]XIAO Luwei, GU Zhenyu. Method and application of patent map[M].Shanghai Jiao Tong University press:HAN Jianmin,2011.1-564(in Chinese).

[4]WU Mengchun, DING lan. Development and application of HighCharts component in the meteorological service[J]. Computer \& Network,2014(12):65-68(in Chinese).

[5]WANG Yong, XU Zhongtao, WANG Ying. Research and implementation of competitive intelligence system based on big data[J].Journal of Guangdong University of Technology,2014,31(3):27-31(in Chinese).

[6]WANG Xingwang, SUN Jiqing. A comparative study on the domestic application of patent map technology[J].JOURNAL OF INFORMATION,2007,26(8):113-119(in Chinese). 